PROCEEDINGS OF THE

AMERICAN MATHEMATICAL SOCIETY

Volume 135, Number 3, March 2007, Pages 787-793

S 0002-9939(06)08531-5

Article electronically published on September 11, 2006

\title{
ON MRA RIESZ WAVELETS
}

\author{
R. A. ZALIK
}

(Communicated by David R. Larson)

\begin{abstract}
We investigate the properties of univariate MRA Riesz wavelets. In particular we obtain a generalization to semiorthogonal MRA wavelets of a well-known representation theorem for orthonormal MRA wavelets.
\end{abstract}

In what follows $\mathbb{Z}$ will denote the integers and $\mathbb{R}$ the real numbers; $t$ and $x$ will always denote real variables. The Fourier transform of a function $f$ will be denoted by $\widehat{f}$. If $f \in L(\mathbb{R})$,

$$
\widehat{f}(x):=\int_{\mathbb{R}} e^{-t x i} f(t) d t .
$$

Let $\mathbb{H}$ be a (separable) Hilbert space with inner product $\langle\cdot, \cdot\rangle$ and norm $\|\cdot\|:=$ $\langle\cdot, \cdot\rangle^{1 / 2}$. A sequence $\left\{f_{k}, k \in \mathbb{Z}\right\} \subset \mathbb{H}$ is called a frame if there are constants $0<A \leq B$ such that for every $f \in \mathbb{H}$

$$
A\|f\|^{2} \leq \sum_{k \in \mathbb{Z}}\left|\left\langle f, f_{k}\right\rangle\right|^{2} \leq B\|f\|^{2} .
$$

The constants $A$ and $B$ are called (lower and upper) bounds of the frame. If only the right-hand inequality in the preceding displayed formula is satisfied for all $f \in \mathbb{H}$, then $\left\{f_{k}, k \in \mathbb{Z}\right\}$ is called a Bessel sequence with bound $B$. A sequence $\left\{f_{k}, k \in \mathbb{Z}\right\} \subset \mathbb{H}$ is called a Riesz basis if its linear span is dense in $\mathbb{H}$ and there are constants $A$ and $B, A>0$, such that for every sequence $\left\{c_{k}, k \in \mathbb{Z}\right\} \subset \ell^{2}$,

$$
A \sum_{k \in \mathbb{Z}}\left|c_{k}\right|^{2} \leq\left\|\sum_{k \in \mathbb{Z}} c_{k} f_{k}\right\|^{2} \leq B \sum_{k \in \mathbb{Z}}\left|c_{k}\right|^{2} .
$$

The constants $A$ and $B$ are called (lower and upper) bounds of the Riesz basis. Every Riesz basis is a frame, every orthonormal basis is a Riesz basis with bounds $A=B=1$, and Riesz bounds and frame bounds coincide. A sequence $\left\{f_{k}, k \in\right.$ $\mathbb{Z}\} \subset \mathbb{H}$ is a Riesz basis if and only if it is a frame having the additional property that upon the removal of any element from the sequence, it ceases to be a frame 1, 2, 7 .

In this paper the underlying Hilbert space will be $L^{2}(\mathbb{R})$ with the usual inner product and norm, and we will study binary affine sequences generated by a single function, i.e. sequences of the form $\left\{\psi_{j, k} ; j, k \in \mathbb{Z}\right\}$, where $\psi \in L^{2}(\mathbb{R})$ and

Received by the editors January 30, 2004 and, in revised form, October 13, 2005.

2000 Mathematics Subject Classification. Primary 42C40.

Key words and phrases. Bessel wavelets, frame wavelets, Riesz wavelets, semiorthogonal wavelets, multiresolution analysis.

The author is grateful to Alfredo L. González and David R. Larson for their helpful comments. 
$\psi_{j, k}(t):=2^{j / 2} \psi\left(2^{j} t-k\right)$. A function $\psi \in L^{2}(\mathbb{R})$ will be called a frame wavelet, a Bessel wavelet, a Riesz wavelet, or an orthonormal wavelet if the affine sequence $\left\{\psi_{j, k} ; j, k \in \mathbb{Z}\right\}$ it generates is respectively a frame, a Bessel sequence, a Riesz basis, or an orthonormal basis of $L^{2}(\mathbb{R})$. When we refer to the bounds of a wavelet $\psi$ we mean the frame, Bessel, or Riesz bounds, as the case may be, of the affine sequence generated by $\psi$. A Riesz wavelet $\psi$ is said to be semiorthogonal if for every $j, k, \ell, m \in \mathbb{Z}$, whenever $j \neq \ell$,

$$
\left\langle\psi_{j, k}, \psi_{\ell, m}\right\rangle=0 .
$$

A multiresolution analysis (MRA) is a sequence $\left\{V_{j} ; j \in \mathbb{Z}\right\}$ of closed linear subspaces of $L^{2}(\mathbb{R})$ such that:

(i) $\quad V_{j} \subset V_{j+1}$ for every $j \in \mathbb{Z}$.

(ii) $\quad$ For every $j \in \mathbb{Z}, f(t) \in V_{j}$ if and only if $f(2 t) \in V_{j+1}$.

(iii) $\quad \bigcup_{j \in \mathbb{Z}} V_{j}$ is dense in $L^{2}(\mathbb{R})$.

(iv) There is a function $\varphi$ such that $\{\varphi(t-k) ; k \in \mathbb{Z}\}$ is an orthonormal basis of $V_{0}$.

It follows from the preceding definition that there is a $2 \pi$-periodic function $p \in L^{2}[0,2 \pi]$ such that

$$
\widehat{\varphi}(2 x)=p(x) \widehat{\varphi}(x) \quad \text { a.e. }
$$

The function $\varphi$ is called a scaling function for the MRA, and $p$ is called the low pass filter associated with $\varphi[\underline{6}$, p. 53].

By $W_{r}$ we will denote the orthogonal complement of $V_{r}$ in $V_{r+1}$. Thus, $V_{r+1}=$ $V_{r} \oplus W_{r}$.

The following results will be used in the sequel.

Theorem A ([6, p. 57]). If $\varphi$ is a scaling function for an $M R A\left\{V_{j} ; j \in \mathbb{Z}\right\}$ and $p$ is the associated low pass filter, then $h$ is an orthonormal wavelet in $W_{0}$ if and only if there is a measurable unimodular and $2 \pi$-periodic function $\nu(x)$, such that

$$
\widehat{h}(2 x)=e^{i x} \nu(2 x) \overline{p(x+\pi)} \widehat{\varphi}(x) \quad \text { a.e. }
$$

Theorem B ([6, pp. 49-50]). Let $\varphi \in L^{2}(\mathbb{R})$ and constants $0<A \leq B$ be given. Then the sequence $\{\varphi(x-k) ; k \in \mathbb{Z}\}$ is a Riesz basis of the closure of its linear span and has bounds $A$ and $B$, if and only if

$$
A \leq \sum_{k \in \mathbb{Z}}|\widehat{\varphi}(x+2 k \pi)|^{2} \leq B \quad \text { a.e. }
$$

In particular, $\{\varphi(x-k) ; k \in \mathbb{Z}\}$ is an orthonormal basis of the closure of its linear span if and only if

$$
\sum_{k \in \mathbb{Z}}|\widehat{\varphi}(x+2 k \pi)|^{2}=1 \quad \text { a.e. }
$$

Let $\psi$ be a frame wavelet in $L^{2}(\mathbb{R})$; for $j \in \mathbb{Z}$, let $P_{j}$ denote the closure of the linear span of $\left\{\psi_{j, k} ; k \in \mathbb{Z}\right\}$, and let $V_{j}:=\sum_{r<j} P_{r}$. Note that $\psi \in V_{1}$. We say that $\psi$ is associated with an MRA, or that $\psi$ is an MRA wavelet, if $M:=\left\{V_{j} ; j \in \mathbb{Z}\right\}$ is a multiresolution analysis; we also say that $\psi$ is associated with $M$.

We begin the statement and proof of our results with the following proposition, of some independent interest. 
Proposition 1. Let $v \in L^{2}(\mathbb{R})$ and let $\mu(x)$ be a measurable $2 \pi$-periodic function. Let $\psi$ be a function such that

$$
\widehat{\psi}(x)=\mu(x) \widehat{v}(x) \quad \text { a.e. }
$$

Then:

(a) If there are constants $B, D>0$ such that $|\mu(x)|^{2} \leq B$ a.e., and $v$ is a Bessel wavelet with bound $D$, then $\psi$ is a Bessel wavelet with bound $B D$.

(b) If there are constants $0<A \leq B, 0<C \leq D$ such that $A \leq|\mu(x)|^{2} \leq B$ a.e., and $v$ is a frame wavelet with bounds $C$ and $D$, then $\psi$ is a frame wavelet with bounds $A C$ and $B D$.

Proof. We will prove (a) only. The proof of (b) will then reduce to finding the lower bound, which is done in a similar way.

Let $f \in L^{2}(\mathbb{R})$. By periodization, as in e.g. [4. p. 270, (2.2)], we have

$$
\sum_{j, k \in \mathbb{Z}}\left|\left\langle f, v_{j, k}\right\rangle\right|^{2}=\frac{1}{2 \pi} \sum_{j \in \mathbb{Z}} 2^{j} \int_{0}^{2 \pi}\left|\sum_{r \in \mathbb{Z}} \widehat{f}\left(2^{j}(x+2 r \pi)\right) \overline{\widehat{v}(x+2 r \pi)}\right|^{2} d x .
$$

Applying (3), we also have

$$
\begin{aligned}
\sum_{j, k \in \mathbb{Z}}\left|\left\langle f, \psi_{j, k}\right\rangle\right|^{2} & =\frac{1}{2 \pi} \sum_{j \in \mathbb{Z}} 2^{j} \int_{0}^{2 \pi}\left|\sum_{r \in \mathbb{Z}} \widehat{f}\left(2^{j}(x+2 r \pi)\right) \overline{\widehat{\psi}(x+2 r \pi)}\right|^{2} d x \\
& =\frac{1}{2 \pi} \sum_{j \in \mathbb{Z}} 2^{j} \int_{0}^{2 \pi}\left|\overline{\mu(x)} \sum_{r \in \mathbb{Z}} \widehat{f}\left(2^{j}(x+2 r \pi)\right) \overline{\widehat{v}(x+2 r \pi)}\right|^{2} d x \\
& \leq B \frac{1}{2 \pi} \sum_{j \in \mathbb{Z}} 2^{j} \int_{0}^{2 \pi}\left|\sum_{r \in \mathbb{Z}} \widehat{f}\left(2^{j}(x+2 r \pi)\right) \overline{\widehat{v}(x+2 r \pi)}\right|^{2} d x .
\end{aligned}
$$

Since

$$
\sum_{j, k \in \mathbb{Z}}\left|\left\langle f, v_{j, k}\right\rangle\right|^{2} \leq D\|f\|^{2},
$$

the assertion follows from (3).

We now turn to Riesz wavelets.

Proposition 2. Let $\mu(x)$ be a measurable $2 \pi$-periodic function, and let $v \in L^{2}(\mathbb{R})$ be a Riesz wavelet with bounds $0<A \leq B$. If $\psi$ is defined by (2) and there are constants $C$ and $D, 0<C<|D| \sqrt{A / B}$ such that

$$
|\mu(x)-D| \leq C \quad \text { a.e. }
$$

then $\psi$ is a Riesz wavelet.

Proof. Let $u:=\psi-D v$. From (2), $\widehat{u}(x)=(\mu(x)-D) \widehat{v}(x)$. Since $v$ is a Riesz wavelet with upper bound $B$, applying Proposition 1 we conclude that $u$ is a Bessel wavelet with bound $C^{2} B<|D|^{2} A$. Since $D v$ is a Riesz wavelet with lower bound $|D|^{2} A$, the assertion follows from [5, Theorem 5].

Theorem 1. Let $M:=\left\{V_{j} ; j \in \mathbb{Z}\right\}$ be an $M R A$, and assume that $\psi \in V_{j}$ for some $j \leq 0$. Then $\psi$ cannot be a Riesz wavelet. 
Proof. We proceed by contradiction. Assume $\psi$ is a Riesz wavelet in $V_{j}$ with bounds $0<A \leq B$, and let $\varphi$ be a scaling function for $M$ with associated low pass filter $p$. Since $\psi \in V_{0}$, there is a $2 \pi$-periodic function $s(x)$ such that

$$
\widehat{\psi}(x)=s(x) \widehat{\varphi}(x) \quad \text { a.e. }
$$

This implies that

$$
\sum_{k \in \mathbb{Z}}|\widehat{\psi}(x+2 k \pi)|^{2}=|s(x)|^{2} \sum_{k \in \mathbb{Z}}|\widehat{\varphi}(x+2 k \pi)|^{2} \quad \text { a.e. }
$$

whence from Theorem B we conclude that

$$
A \leq|s(x)|^{2} \leq B \quad \text { a.e. }
$$

Moreover, the well-known identity

$$
|p(x)|^{2}+|p(x+\pi)|^{2}=1
$$

implies that $|p(x)| \leq 1$. Thus, if

$$
m(x):=\frac{s(2 x) p(x)}{s(x)},
$$

we infer that $|m(x)|^{2} \leq B / A$. Since

$$
\widehat{\psi}(2 x)=s(2 x) p(x) \widehat{\varphi}(x)=m(x) \widehat{\psi}(x)
$$

and $m \in L^{2}(0,2 \pi)$ and is $2 \pi$-periodic, we conclude that $\psi(t / 2)$ is in the closure of the linear span of the sequence $\{\psi(t-k) ; k \in \mathbb{Z}\}$. This is incompatible with the assumption that $\left\{\psi_{j, k} ; j, k \in \mathbb{Z}\right\}$ is a Riesz basis, and we have reached a contradiction.

Corollary 1. Let $M:=\left\{V_{j} ; j \in \mathbb{Z}\right\}$ be an MRA. If $j \neq 0$ there is no function $u$ such that $\{u(t-k) ; k \in \mathbb{Z}\}$ is a Riesz basis of $V_{j}$.

Proof. If $\{u(t-k) ; k \in \mathbb{Z}\}$ is a Riesz basis of $V_{j}$, then there is a function $v$ such that $\{v(t-k) ; k \in \mathbb{Z}\}$ is an orthonormal basis of $V_{j}$ [6, pp. 48-51]. Setting $U_{r}:=V_{r+j}$ we see that $\left\{U_{r} ; r \in \mathbb{Z}\right\}$ is an MRA. If $W_{0}^{\prime}$ denotes the orthogonal complement of $U_{0}$ in $U_{1}$, then there is an orthonormal (and therefore Riesz) wavelet $h^{\prime}$ in $W_{0}^{\prime} \subset$ $U_{1}=V_{j+1}$. If $j<0$ this implies that $V_{0}$ contains an orthonormal wavelet, which is a contradiction. On the other hand if $j>0$, let $h$ be an orthonormal wavelet in $W_{0}$. Since $W_{0} \subset V_{1}=U_{1-j} \subset U_{0}$, we see that $U_{0}$ contains an orthonormal wavelet, and we have a contradiction in this case as well.

Theorem 1 is supplemented by the following.

Theorem 2. Let $M:=\left\{V_{j} ; j \in \mathbb{Z}\right\}$ be an $M R A$, and assume that $\psi \in W_{r}$ for some $r \neq 0$. Then $\psi$ cannot be a Riesz wavelet.

Proof. Since

$$
V_{0}=\bigoplus_{j<0} W_{j}
$$

the assertion for $r<0$ follows from Theorem 1. Assume now that $r>0$. Let $h \in W_{0}$ be an orthonormal wavelet, and let $\psi$ be a Riesz wavelet in $W_{r}$ with bounds $A$ and $B$. Then $\psi_{-r, 0} \in W_{0}$. From, e.g., [6. p. 57, Lemma 2.11], we know that $W_{0}$ is closed under integral translations (this can also be easily obtained from the definition of $\left.W_{0}\right)$. Since $r>0$, we deduce that $\psi_{-r, k} \in W_{0}$ for every $k \in \mathbb{Z}$. Thus $\psi_{j, k} \in W_{j+r}$ for all $j, k \in \mathbb{Z}$. 
Let $f \in W_{r}$. The hypotheses imply there is a sequence $\left\{d_{j, k} ; j, k \in \mathbb{Z}\right\} \in \ell^{2}\left(\mathbb{Z}^{2}\right)$ such that $f=\sum_{j, k \in \mathbb{Z}} d_{j, k} \psi_{j, k}$ (in the $L^{2}$ sense). Define $f_{1}:=\sum_{k \in \mathbb{Z}} d_{0, k} \psi_{0, k}$ and $f_{2}=f-f_{1}$. Since $f-f_{1} \in W_{r}$ and $f_{2} \in W_{r}^{\perp}$, we conclude that $f_{2}=0$; thus, $\overline{\operatorname{span}\{\psi(\cdot-k) ; k \in \mathbb{Z}\}}=W_{r}$. Since $h_{r, 0} \in W_{r}$, this implies that there is a $2 \pi-$ periodic function $m(x)$ such that

$$
\widehat{h_{r, 0}}(x)=m(x) \widehat{\psi}(x) \quad \text { a.e. }
$$

Thus Theorem B implies that

$$
\begin{aligned}
2^{-r}=2^{-r} \sum_{k \in \mathbb{Z}}\left|\widehat{h}\left(2^{-r} x+2 k \pi\right)\right|^{2} & \\
& =|m(x)|^{2} \sum_{k \in \mathbb{Z}}\left|\widehat{\psi}\left(x+2^{r+1} k \pi\right)\right|^{2} \leq|m(x)|^{2} B \text { a.e. }
\end{aligned}
$$

On the other hand, since

$$
\sum_{k \in \mathbb{Z}}\left|\widehat{h}\left(x+2^{1-r} k \pi\right)\right|^{2}=\sum_{\ell=0}^{2^{r}-1} \sum_{n \in \mathbb{Z}}\left|\widehat{h}\left(x+2^{1-r} \ell \pi+2 n \pi\right)\right|^{2}=2^{r} \quad \text { a.e. }
$$

we see that

$$
\begin{aligned}
1=2^{-r} \sum_{k \in \mathbb{Z}}\left|\widehat{h}\left(2^{-r} x+2^{1-r} k \pi\right)\right|^{2} & \\
& =|m(x)|^{2} \sum_{k \in \mathbb{Z}}|\widehat{\psi}(x+2 k \pi)|^{2} \geq|m(x)|^{2} A \quad \text { a.e. }
\end{aligned}
$$

We therefore conclude that

$$
2^{-r} B^{-1} \leq|m(x)|^{2} \leq A^{-1} \quad \text { a.e. }
$$

and Proposition 1 implies that $h_{r, 0}$ is a frame wavelet. This implies that

$$
\left\{2^{j / 2} h\left(2^{j} t-2^{r} k\right) ; j, k \in \mathbb{Z}\right\}
$$

is a frame wavelet. Since

$$
\left\{2^{j / 2} h\left(2^{j} t-k\right) ; j, k \in \mathbb{Z}\right\}
$$

is a Riesz basis by hypothesis and (4) is a subsequence of (5), this is incompatible with the definition of Riesz basis, and we have obtained a contradiction.

We are now ready to prove

Theorem 3. Let $\varphi$ be a scaling function for an $M R A M=\left\{V_{j} ; j \in \mathbb{Z}\right\}$, and let $p$ be the associated low pass filter. The following propositions are equivalent: $B$.

(a) $\psi$ is a semiorthogonal Riesz wavelet associated with $M$ with bounds $0<A \leq$

(b) There is a measurable $2 \pi$-periodic function $\mu(x)$ such that

$$
\widehat{\psi}(2 x)=e^{i x} \mu(2 x) \overline{p(x+\pi)} \widehat{\varphi}(x) \quad \text { a.e. }
$$

and

$$
A \leq|\mu(x)|^{2} \leq B \quad \text { a.e. }
$$


Proof. The definitions imply that $\psi$ is a semiorthogonal wavelet if and only if $\psi \in W_{0}$. Let

$$
r(x):=\left(\sum_{k \in \mathbb{Z}}|\widehat{\psi}(x+2 k \pi)|^{2}\right)^{1 / 2}
$$

and

$$
\widehat{h}(x):=\widehat{\psi}(x) / r(x) .
$$

Then, as remarked in [3, p. 78], $h$ is an orthonormal wavelet. Since $1 / r(x)$ is $2 \pi-$ periodic and bounded, it follows that $h \in W_{0}$. Thus $h$ has a representation of the form (11). Setting $\mu(x):=r(x) \nu(x)$, we readily see that (6) and (7) are satisfied.

Conversely, assume there is a $2 \pi$-periodic function $\mu(x)$ such that (6) and (7) are satisfied. Let

$$
\operatorname{sign} \mu(x):= \begin{cases}\mu(x) /|\mu(x)| & \text { if } \mu(x) \neq 0 \\ 1 & \text { if } \mu(x)=0\end{cases}
$$

Setting

$$
\widehat{h}_{1}(2 x):=e^{i x} \operatorname{sign}(\mu(x)) \overline{p(x+\pi)} \widehat{\varphi}(x),
$$

we conclude from Theorem A that $h_{1}$ is an orthonormal wavelet. Moreover, (6) implies that

$$
\widehat{\psi}(x)=|\mu(x)| \widehat{h}_{1}(x) \quad \text { a.e. }
$$

Since

$$
|| \mu(x)|-\sqrt{B}| \leq \sqrt{B}-\sqrt{A}<\sqrt{B} \quad \text { a.e., }
$$

we deduce from Proposition 2 that $\psi$ is a Riesz wavelet. Indeed, since $A^{\prime}=1$ and $B^{\prime}=1$ are Riesz bounds for $h_{1}$, setting $C=\sqrt{B}-\sqrt{A}$ and $D=\sqrt{B}$, we see that $0<C<|D| \sqrt{A^{\prime} / B^{\prime}}$ and

$$
|| \mu(x)|-D| \leq C \quad \text { a.e. }
$$

Proposition 2 does not yield sufficiently accurate information about the Riesz bounds of $\psi$. However, from Proposition 1 we readily see that $\psi$ is a frame wavelet with lower bound $A$ and upper bound $B$. Since frame bounds and Riesz bounds coincide, we conclude that $A$ and $B$ are a lower and an upper Riesz bound of $\psi$, respectively.

Clearly Theorem A is a particular case of Theorem 3. From Theorem A and Theorem 3 we obtain

Corollary 2. A function $\psi$ is a semiorthogonal MRA Riesz wavelet with bounds $0<A \leq B$ if and only if there is a measurable $2 \pi$-periodic function $\mu(x)$ that satisfies (77), and an MRA orthonormal wavelet $h$, such that

$$
\widehat{\psi}(x)=\mu(x) \widehat{h}(x) \quad \text { a.e. }
$$

\section{REFERENCES}

1. J. J. Benedetto and D. F. Walnut, Gabor frames for $L^{2}$ and related spaces, in "Wavelets: Mathematics and Applications" (J. J. Benedetto and M. W. Frazier, Eds.), pp. 97-162, CRC Press, Boca Raton, FL, 1994. MR1247515 (94i:42040)

2. O. Christensen, "An Introduction to Frames and Riesz Bases", Birkhäuser, Boston, 2003. MR1946982(2003k:42001)

3. C. K. Chui, "An Introduction to Wavelets," Academic Press, San Diego, 1992. MR:1150048 (93f:42055) 
4. C. K. Chui and X. Shi, Inequalities of Littlewood-Paley type for frames and wavelets, SIAM J. Math. Anal. 24 (1993), 263-277. MR1199539 (94d:42039)

5. S. J. Favier and R. A. Zalik, On the stability of frames and Riesz bases, Appl. Comput. Harm. Anal. 2 (1995), 160-173. MR 1325538 (96e:42030)

6. E. Hernández and G. Weiss, "A First Course on Wavelets", CRC Press, Boca Raton, FL, 1996. MR.1408902 (97i:42015)

7. R. M. Young, "An Introduction to Nonharmonic Fourier Series", Revised $1^{\text {st }}$ ed., Academic Press, San Diego, 2002. MR1836633 (2002b:42001)

Department of Mathematics and Statistics, Auburn University, Auburn, Alabama $36849-5310$

E-mail address: zalik@auburn.edu 